\title{
Aplicación de dispositivos electrónicos para el ahorro de energía eléctrica, en la materia de Instrumentación apoyando la docencia y aprendizaje
}

\section{Applying electronical devices to save electrical energy in the subject of Instrumentation, supporting teaching and learning} GALARZA-SOSA, Oscar†*, MALDONADO-PESINA, Ericka, MARTINEZ-TOVAR, Cesar y
GALINDO-CERDA, Herlinda

Instituto Tecnológico de Linares / Tecnológico Nacional de México

ID $1^{\text {er }}$ Autor: Oscar, Galarza-Sosa / ORC ID: 0000-0002-1036-4399, CVU CONACYT ID: 640809

ID $1^{\text {er }}$ Coautor: Ericka, Maldonado-Pesina / ORC ID: 0000-0003-0926-5542, CVU CONACYT ID: 519549

ID $2^{\text {do }}$ Coautor: Cesar, Martinez-Tovar / ORC ID: 0000-0002-0684-2511, CVU CONACYT ID: 1014345

ID $3^{\text {er }}$ Coautor: Herlinda, Galindo-Cerda / ORC ID: 0000-0003-0889-0119, CVU CONACYT ID: 640811

DOI: $10.35429 /$ JTAE.2019.10.3.29.35

Recibido: 30 de Agosto, 2019; Aceptado 30 de Diciembre 2019

Resumen

Los dispositivos electrónicos a escala utilizados para crear un sistema automatizado que permita el control de encendido y apagado autónomo de luces y aire acondicionado, según requerimientos dentro de un edificio con el fin de hacer más eficiente el consumo de energía eléctrica. Mediante una estructura aplicada en la docencia y aprendizaje que mejore la asimilación de los conceptos básicos y obtener conocimientos sobre el enfoque de sistemas electromecánicos, que permita un mejor análisis para la toma de decisiones, búsqueda de alternativas y oportunidades; para redefinir y solucionar problemas estableciendo una relación armónica con su medio ambiente comprendiendo todas las entradas, salidas, sus interrelaciones y características de sus elementos permitiendo modelar un sistema de interés con tarjeta arduino para obtener una solución viable con el empleo de múltiples dispositivos. En el entorno industrial y educativo que existe se tiene la necesidad de contar con los dispositivos de control que permitan, una forma de enseñanza educativa proactiva entre los alumnos innovando un instrumento electrónico, flexible y actualizado con el cual se puedan desarrollar proyectos que estén alineados a los requerimientos de calidad a favor del medio ambiente.

Arduino, Electricidad, Electrónico.

\begin{abstract}
The industrial and didactics background, in which we can find the educational institutions, has the neccesity to improve control electronical devices which permit, on one hand, a proactive and productive integral formation of creation and innovation among students, and on the the hand, a flexible electronic device in which they can develop alined projects with up to date requirements, as in the case of automatized systems alined to get an efficient use of electricity. For this case, it is proposed that using these electronical devices, as the Arduino, to create a control sistems that permit to turn on and off the lights or air conditioning systems in a building in an autonomus way to make efficient the electrical consumption. Through an estructurization applied on teaching and learning that improves the asimilation of basical concept's, and to get knowledge on the focus of electromechanical systems that permit a better analysis for taking decisions, searching alternatives and opportunities; redefined and solved problems establishing an armonic relationship with the environment that encompasses inputs,outputs, characteristics and interrelationships among elements permitting to model an interesting electronical system to get a viable solution on scale devices.
\end{abstract}

Arduino, Electricity, Electronic

Citación: GALARZA-SOSA, Oscar, MALDONADO-PESINA, Ericka, MARTINEZ-TOVAR, Cesar y GALINDOCERDA, Herlinda. Aplicación de dispositivos electrónicos para el ahorro de energía eléctrica, en la materia de Instrumentación apoyando la docencia y aprendizaje. Revista de Tecnología y Educación. 2019. 3-10: 29-35

\footnotetext{
* Correspondencia del Autor (Correo electrónico: omgalarza @ hotmail.com)

$\dagger$ Investigador contribuyendo como primer autor.
} 


\section{Introducción}

El entorno industrial y educativo en el que se encuentra inmerso el Instituto Tecnológico de Linares, en especial para la carrera de Ingeniería en Electromecánica, tiene la necesidad de contar con los elementos electrónicos de control que permitan como primer punto, una forma de enseñanza educativa proactiva entre los alumnos, y como segundo punto, un instrumento electrónico, flexible, actualizado y a escala, con el cual se puedan desarrollar proyectos que estén alineados a los requerimientos del ambiente laboral, como es el caso del uso eficiente de la energía eléctrica.

Asimismo, la demanda industrial y el cuidado de las energías es un área con gran crecimiento laboral, por lo que es necesario que los alumnos posean conocimientos sobre esta área, con base en enseñanza teórica practica que le aporten una visión amplia (Torres \& Pérez, 2019).

$\mathrm{Y}$ con base en lo anterior, es un requisito indispensable llevar a cabo proyectos didácticos donde se busque solucionar problemas que se presentan en las empresas, como puede ser mejorar sus sistemas de producción, automatización de proceso, disminuir costos, etc., pero a simple vista se podría decir que son demasiados caros para que los alumnos de nivel superior de un Instituto Tecnológico los realicen. Para tal caso surge la oportunidad de llevar a cabo una propuesta para dichas mejoras con un instrumento muy flexible, y lo más importante económico, como lo son los dispositivos electrónicos de software abierto, por ejemplo, el Arduino.

Y que como señala Rojas \& Contreras (2019), en la enseñanza de nivel superior es transcendental la utilización de elementos que permitan una enseñanza practica en talleres o laboratorios con el fin de que los ingenieros se preparen de forma eficiente y acercarlos al entorno real industrial. La aplicación de estas herramientas, como se ha señalado anteriormente es precisamente el hecho de que son baratos, y sus componentes de desarrollo (sensores, actuadores, etc.) son perfectamente adaptables, dando acceso a desarrollar prototipos didácticos a escala, y lo más importantes, que estos pueden ser una posible propuesta de implementación real en la industria actual ya con elementos profesionales.
Asimismo, es transcendental indicar que la utilización de los dispositivos electrónicos a escala, además de dar acceso a encontrar soluciones innovadoras y baratas a problemáticas existentes en una empresa, sin importar su giro, como es el caso del ahorro de energía eléctrica, permite desarrollar un proceso educativo integral para los alumnos, ya que posee un ambiente de programación simple y fácil de usar para cualquier estudiante y docente.

Por lo tanto, se plantea la construcción de un control automático de encendido y apagado de luces y aire acondicionados según exigencias de una oficina o edificio con base en el uso de dispositivos electrónicos, lo que permitirá obtener de ahorro de energía eléctrica y al mismo tiempo desarrollar un proceso educativo integral que cumpla con las exigencias del nivel superior tecnológico de nuestros alumnos.

Rivera (2019), menciona que el Método de Proyectos encamina al estudiante a elaborar de forma organizada y participativa actividades, que le dan acceso no solo a encontrar y estar al tanto de nuevos procesos, sino además experimentar el gusto de un resultado palpable por el mismo.

\section{Objetivos}

\section{Objetivo General}

Utilizar dispositivos electrónicos a escala con los dispositivos electrónicos para crear un sistema automatizado, que permita el control de encendido y apagado autónomo de luces y aire acondicionado según requerimientos dentro de un edificio con el fin de hacer más eficiente el consumo de energía eléctrica, de la misma forma, lograr que los alumnos de la materia de Instrumentación establezcan de manera correcta la clasificación, empleo, manipulación, mantenimiento y calibración de los controladores PID (Proporcional - Integral Derivativo), con el fin de mejorar la asimilación integral de los conocimientos teóricos prácticos de la asignatura.

\section{Objetivos Específicos}

a. Crear un sistema automático con base en un dispositivo electrónico para ahorrar energía eléctrica de un edificio a partir de controlar el encendido y apagado de luces y aire acondicionado según convenga.

GALARZA-SOSA, Oscar, MALDONADO-PESINA, Ericka, MARTINEZ-TOVAR, Cesar y GALINDO-CERDA, Herlinda. Aplicación de dispositivos electrónicos para el ahorro de energía eléctrica, en la materia de Instrumentación apoyando la docencia y aprendizaje. Revista de Tecnología y Educación. 2019 
b. Desarrollar una estructura de docencia y aprendizaje para la materia de Instrumentación que mejore la asimilación de los conceptos básicos y obtener conocimientos sobre el enfoque de sistemas electromecánicos, que le permita un mejor análisis para la toma de decisiones, búsqueda de alternativas y oportunidades, para redefinir y solucionar problemas, estableciendo una relación armónica con su medio ambiente comprendiendo todas las entradas, salidas del sistema, sus interrelaciones y características de sus elementos permitiéndole modelar un sistema de interés para obtener una solución viable con el empleo de dispositivos electrónicos.

c. Incrementar en un $15 \%$ la calificación final de los alumnos de la materia de Instrumentación.

\section{Limitaciones}

Desde el punto de vista docente, se puede especular que cualquier desarrollo o aplicación metodológico que pretenda mejorar un aprendizaje significativo e integral, presente imperfecciones relacionadas con el entorno cultural y socioeconómico, dado que es imposible establecer una coherencia de aprendizaje estándar para los alumnos de nivel superior en México.

Por lo que se tiene que tomar en cuenta necesariamente que, aunque los dispositivos electrónicos flexibles a escala se pueden considerar económicos, en algunas regiones de nuestro país, en otras partes, puede suceder lo contrario, y este punto sería trascendental para ser considerado.

Por lo cual se debería ser consiente que al desarrollar esta propuesta tal vez sea necesario implementar trabajo en equipo de los estudiantes y docentes, contemplando un trabajo multidisciplinario en academias, para buscar que sea aún más económico el llevar a cabo el proyecto final.

\section{Antecedentes}

Reyes (2015), señala que el Arduino es una plataforma electrónica y de programación en diseño abierto con una amplia gama de aplicaciones en ciencias exactas y principalmente en ingenierías, además menciona que actualmente, el Arduino se ha posicionado de forma vertiginosa como una eficaz herramienta tecnológica en la industria, universidades y centros de investigación, debido a su propuesta de arquitectura abierta que lo hace un sistema por sí mismo, para llevar a cabo una automatización de procesos físicos "a la medida".

Por ende, el Arduino brinda a los investigadores, profesores, ingenieros $\mathrm{y}$ estudiantes un soporte de desarrollo tecnológico con una gran diversidad de aplicaciones. Y particularmente en el área de Docencia, el Arduino se ha transformado en una herramienta pedagógica muy potente e integral, que mejora el proceso de transferencia de conocimientos en los cursos de microcontroladores, instrumentación, control, servomecanismos, sistemas dinámicos lineales y no lineales, robótica, física, circuitos eléctricos, etc. (Palacios, 2015). López (2016), afirma que los Arduino son una plataforma hardware libre proyectada especialmente con una electrónica fundamentada en una placa, un micro controlador y un entorno de desarrollo que da acceso a cualquier persona, realizar cualquier tipo de proyecto multidisciplinar. Con base en que puede ser utilizada para diseñar y fabricar dispositivos autónomos, ligeros de tamaño y de costo mínimo; con amplias posibilidades de conectividad y aplicación.

Los controladores PID son ampliamente utilizados en el monitoreo de variables esenciales dentro de la industria. Así, que, desde el punto de vista moderno, un controlador PID permite controlar señales de segundo orden con una integración, es decir, se ajustan en términos de sus componentes de proporción y derivación, lo que da acceso a obtener resultados sobresalientes en aplicaciones de rastreo de variables. El concepto Integral (I) permite una corrección proporcional completa del error, esta maniobra tiene el propósito de reducir el error. El concepto Derivativo (D) permite lograr propiedades predictivas a la actuación de la Integral, generando un control Proporcional (P) del error detectado (V.V.A.A., 2011). 
Asimismo, Ogata (2003), indica que la mitad de los controladores usados en la actualidad en la industria poseen un sistema de control PID o PID modificado. El Arduino es una placa de hardware libre que concentra un microcontrolador reprogramable y una serie de pines hembra, los cuales están conectados al microcontrolador, y que permiten conectar de manera sencilla y fácil una gran cantidad de sensores y actuadores (Torrente, 2013).

Otra característica notable de este dispositivo electrónico, es que maneja un hardware y software de código abierto, lo que le permite ser una excelente opción para adaptarlo a cualquier necesidad de requerida desde el punto de vista educativo y de desarrollo en ingeniería (Valdés \& Pallás, 2016).

A la vez el uso y aplicación de métodos de programación y adquisición de señales (DAQ) por software y hardware permiten un control óptimo de las variables de entrada y de salida de un cualquier sistema con gran precisión (Bastidas \& Medina, 2019).

\section{Planteamiento del problema}

Al observar los resultados obtenidos en la materia de Instrumentación, se detectó una deficiencia en la asimilación de las competencias que la asignatura proyecta, con base en que los alumnos presentan dificultades en poner en práctica los conocimientos teóricos de la misma y a una auto reflexión crítica.

Posteriormente se llevó a cabo un estudio en conjunto con la academia de IEME del Instituto Tecnológico de Linares, con el fin de detectar un proceso completo que ayudará a los estudiantes de la materia de Instrumentación, resultando en una propuesta de trabajo que incluya la elaboración de un proyecto integrador con elementos electrónicos a escala, para lograr una enseñanza donde se alcance los objetivos planteados por la materia, ya que estos dispositivo son flexibles y económicos para realizar cualquier tipo de prototipo que va en correlación con la asignatura en cuestión.

La aplicación de dispositivos electrónicos para el ahorro de energía eléctrica en la materia de Instrumentación apoyando la docencia y aprendizaje. ¿Podría mejorar el aprendizaje integral de los alumnos de la materia de Instrumentación?
Problemática: Aprendizaje integral con base en la conclusión de un proyecto final.

Factor A (materia): Aplicación de dispositivos electrónicos para el ahorro de energía eléctrica en la materia de Instrumentación, con el propósito de un desarrollo integral de los conocimientos teóricos prácticos apoyando la docencia y aprendizaje de la materia.

Factor B (método): Aplicación de componentes y herramientas electrónicas, considerando manual, semiautomático y automático.

\section{Metodologia}

El procedimiento planteado contempla un proceso de desarrollo completo, para lograr una adecuada interpretación de resultados con respecto a la problemática descubierta, declarado lo anterior, en seguida se indica de forma clara la metodología que se contemplan para llevar a cabo el proyecto de investigación:

El tipo de investigación con base en las variables a estudiar será Explicativa, con el fin de detectar la causa efecto de la situación propuesta. Así mismo, para el diseño de investigación se establecerán estrategias de índole Experimental, considerando como elemento de estudio al grupo de la materia de Instrumentación de la carrera de Ingeniería en Electromecánica para el semestre enero junio del 2019 del Instituto Tecnológico de Linares, el cual estará sometido a la estrategia de proyecto planteada.

Para la población se considerará el total de alumnos que se inscribieron en la materia de Instrumentación de la carrera IEME en los dos últimos semestres del Instituto Tecnológico de Linares.

La muestra principal estará formada por los alumnos que se inscriban en el periodo enero junio del 2019 y del semestre anterior inmediato en la materia de instrumentación del Instituto Tecnológico de Linares, para los cuales se implementará un tipo de muestreo no probabilístico intencional, considerando como criterios: calificación de la materia y calificación de prototipo final.
GALARZA-SOSA, Oscar, MALDONADO-PESINA, Ericka, MARTINEZ-TOVAR, Cesar y GALINDO-CERDA, Herlinda. Aplicación de dispositivos electrónicos para el ahorro de energía eléctrica, en la materia de Instrumentación apoyando la docencia y aprendizaje. Revista de Tecnología y Educación. 2019 
De la misma forma, la técnica seleccionada para la recolección de datos será por medio de la calificación final del proyecto integrador separada en tres secciones de desarrollo (manual, semiautomático y automático), los cuales serán aplicados en los alumnos de la materia de Instrumentación al final del semestre agosto diciembre 2018 y enero junio del 2019 del Instituto Tecnológico de Linares.

Para el análisis de datos, se estableció un estudio estadístico inferencial y descriptivo, con base en el estudio de los resultados de la calificación final de avance de proyecto de la materia y el proyecto final.

El análisis de varianza (ANOVA) es la metodología central en el estudio de datos experimentales. La idea general de esta herramienta es la de separar la variación total en las partes con las que contribuye cada fuente de variación en el experimento a desarrollar (Gutiérrez, 2012).

La meta del análisis de varianza es comprobar la hipótesis de igualdad de los tratamientos con base en la media de la correspondiente variable de respuesta de estudio:

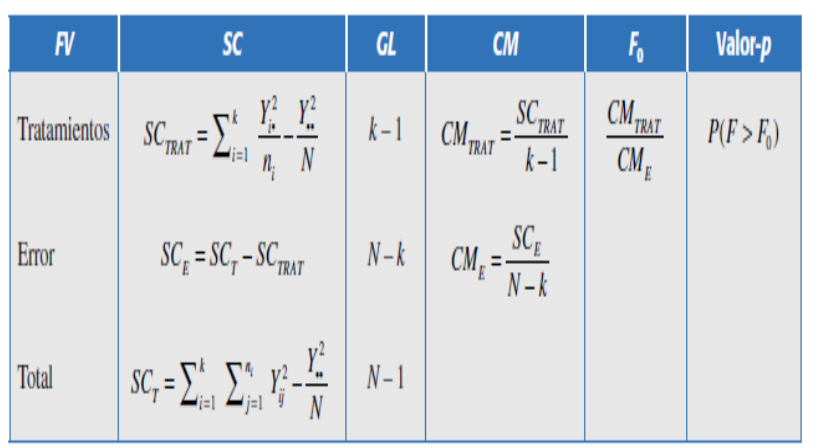

Figura 1 Tabla de ANOVA (Gutiérrez, 2012)

Una vez que se rechazó la hipótesis nula en un análisis de varianza, es obligatorio ir a detalle y ver cuáles tratamientos son desiguales. Enseguida, se muestran algunas técnicas para llevar a cabo dichos tratamientos a más detalle.

a. Método LSD (diferencia mínima significativa), este establece la diferencia mínima que debe presentarse entre dos medias muéstrales con el fin de establecer que los tratamientos estadísticos correspondientes son realmente significativamente desiguales. b. Método de Turkey, esta es una de las técnicas más conservadoras para comparar las medias de un análisis, esta consiste principalmente en comparar las diferencias entre medias muéstrales con el valor critico establecido.

La presente investigación hará uso para el análisis de datos del software Minitab®, el cual es una excelente herramienta tecnológica para llevar a cabo un estudio estadístico de forma profesional.

Ya que este programa permite simplificar enormemente los cálculos previstos para el presente proyecto. (Montgomery, 2013), señala que en muchos experimentos interviene el estudio de dos o más factores. En general, los diseños factoriales son los más eficientes para este tipo de experimentos. Por diseños factoriales se entiende que en cada ensayo o réplica completa del experimento se investigan todas las combinaciones posibles de los niveles de los factores.

\section{Recolección y Análisis de datos}

Se estableció que la población sería el total de alumnos inscritos en los dos últimos semestres de la materia de Instrumentación dentro del Instituto Tecnológico de Linares, es decir, Agosto - Diciembre 2018 y Enero - Junio 2019.

Para el primer grupo se estableció un desarrollo sin el método propuesto, es decir, un proyecto final enfocado en el ahorro de energía sin la aplicación de dispositivos electrónicos a escala (Arduino).

Para el segundo grupo se estableció un desarrollo con el método propuesto del mismo proyecto, es decir, con la aplicación de dispositivos electrónicos a escala (Arduino).

Como siguiente etapa de investigación, enseguida se muestran la recopilación de datos, con base en las calificaciones separadas por las diferentes propuestas de desarrollo de prototipo final de la materia.

Se solicita a los estudiantes la elaboración de un prototipo que se controlado manualmente, en la segunda que el mismo prototipo posea un control semiautomático, y se finaliza con la implementación de un prototipo totalmente automático. 
Cabe aclarar nuevamente, que se solicitó a los estudiantes que el proyecto se orientara al ahorro de energía eléctrica para crear un sistema automatizado que permita el control de encendido y apagado autónomo de luces y aire acondicionado, según requerimientos dentro de un edificio.

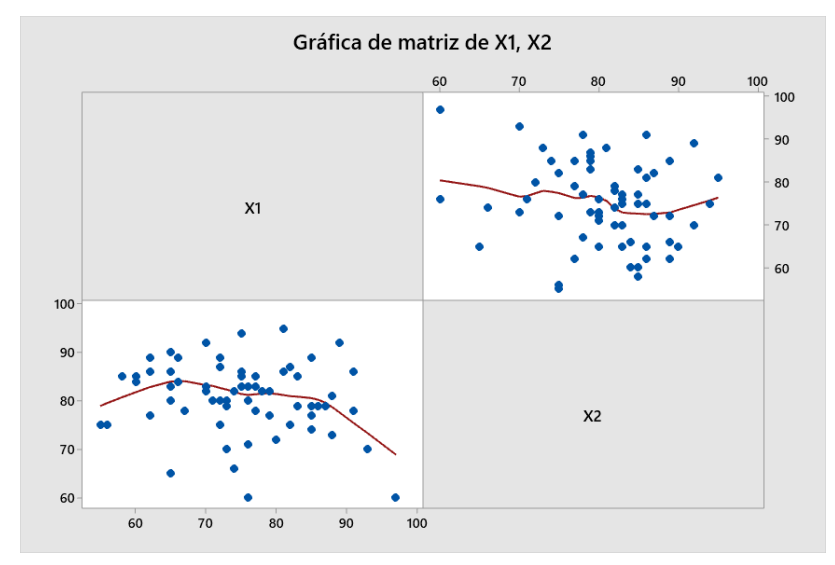

Grafica 1 Correlación de variables X1 y X2

Se pude concluir que proceso de aprendizaje propuesto uniformiza considerablemente las calificaciones de forma positiva, es decir, se presentan valores menores en $\mathrm{X} 1 \mathrm{y}$ tienden a valores mayores en $\mathrm{X} 2$ (calificaciones).

\begin{tabular}{|l|r|r|r|r|r|}
\hline \multicolumn{1}{|c|}{ Variable } & $\mathbf{N}$ & \multicolumn{1}{c|}{ N*dia } & Mediana & Máximo \\
\hline $\begin{array}{l}\text { Agosto-Diciembre } \\
2018\end{array}$ & 25 & 0 & 51.84 & 79.33 & 88.00 \\
\hline Enero-Junio 2019 & 23 & 0 & 72.20 & 81.67 & 91.00 \\
\hline
\end{tabular}

Tabla 1 Valores estadísticos de la muestra

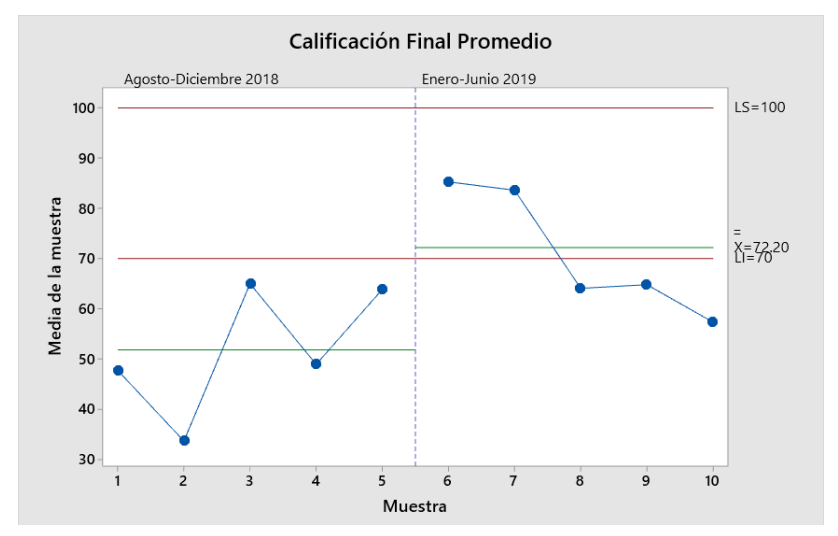

Grafica 2 Antes y después de aplicación de metodología en calificación final promedio

Se observa en la gráfica que las medias de las calificaciones de antes y después de la aplicación de la propuesta aumento desde un 51.84 a 72.20 , lo que representa un incremento promedio de un $20 \%$ en la calificación final, además de mejorar la índice aprobación desde el $64 \%$ hasta el $87 \%$.

\section{Conclusiones}

$\mathrm{Si}$ se utilizan dispositivos y herramientas electrónicas para el ahorro de energía eléctrica de la materia de instrumentación (aprovechamiento) apoyando la docencia y aprendizaje, entonces se mejorará el aprendizaje integral de la asignatura.

La utilización de dispositivos y herramientas (Arduino), mejora en gran medida el aprovechamiento de la materia de instrumentación, y, por ende, es un elemento que apoya considerablemente la docencia y el aprendizaje, además de mejorar el aprendizaje teórico - práctico continúo y permanente.

Y, por último, se puede declarar que el desarrollo, aplicación y la utilización de dispositivos electrónicos, considerando métodos $\mathrm{y}$ aprovechamientos, apoya la docencia y el aprendizaje para la materia de Instrumentación, ya que presentan una correlación positiva que mejora en gran medida la calificación.

Se puede observar con los resultados antes obtenidos que la metodología de trabajo se puede optimizar para una segunda etapa de verificación, ya que los datos obtenidos reflejan información relevante de la actuación de técnicas didácticas que deben ser mejoradas aún más.

Además, cabe señalar que la herramienta, dispositivo electrónico propuesto como lo es el Arduino, es de significancia relevante para la docencia actual, por lo que sugerimos su aplicación efectiva en el actual y futuro modelo educativo.

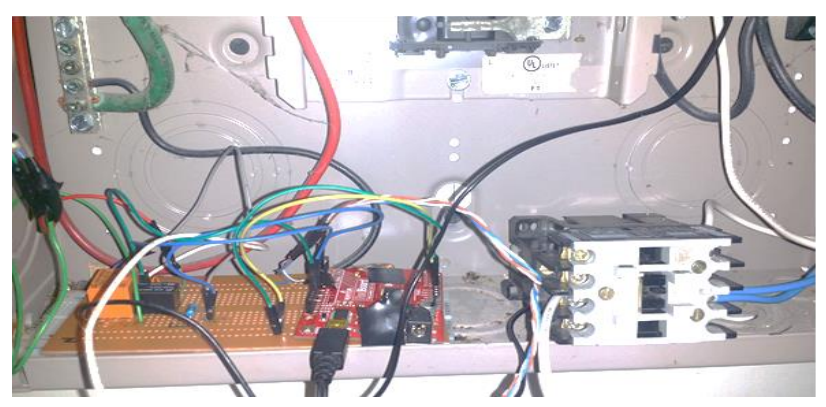

Figura 2 Interconexión Arduino
GALARZA-SOSA, Oscar, MALDONADO-PESINA, Ericka, MARTINEZ-TOVAR, Cesar y GALINDO-CERDA, Herlinda. Aplicación de dispositivos electrónicos para el ahorro de energía eléctrica, en la materia de Instrumentación apoyando la docencia y aprendizaje. Revista de Tecnología y Educación. 2019 


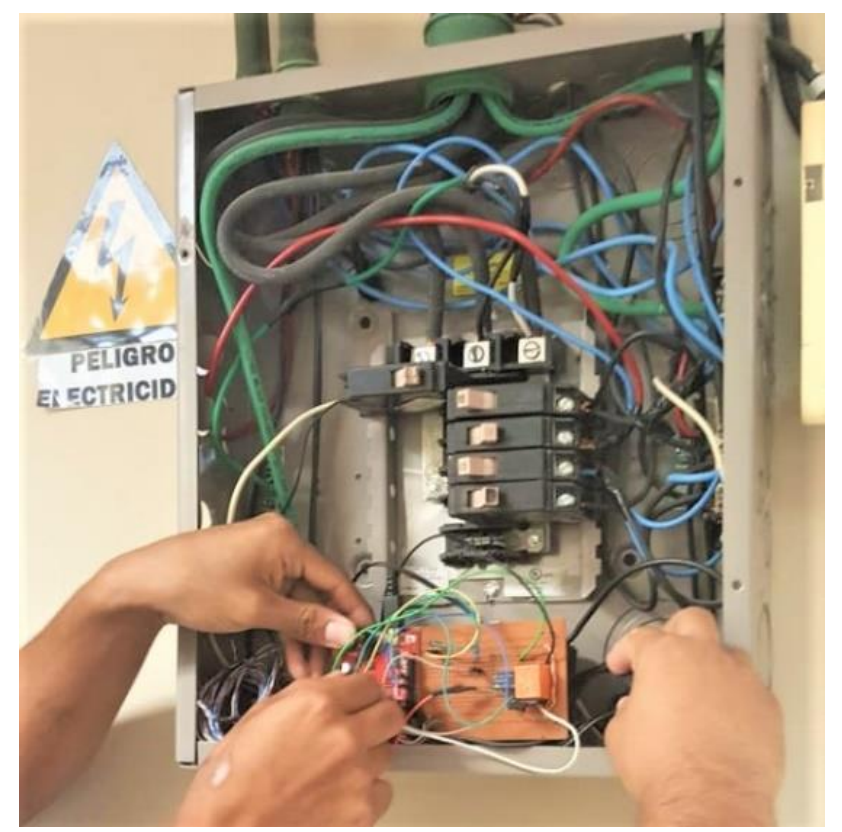

Figura 3 Vista frontal del prototipo terminado

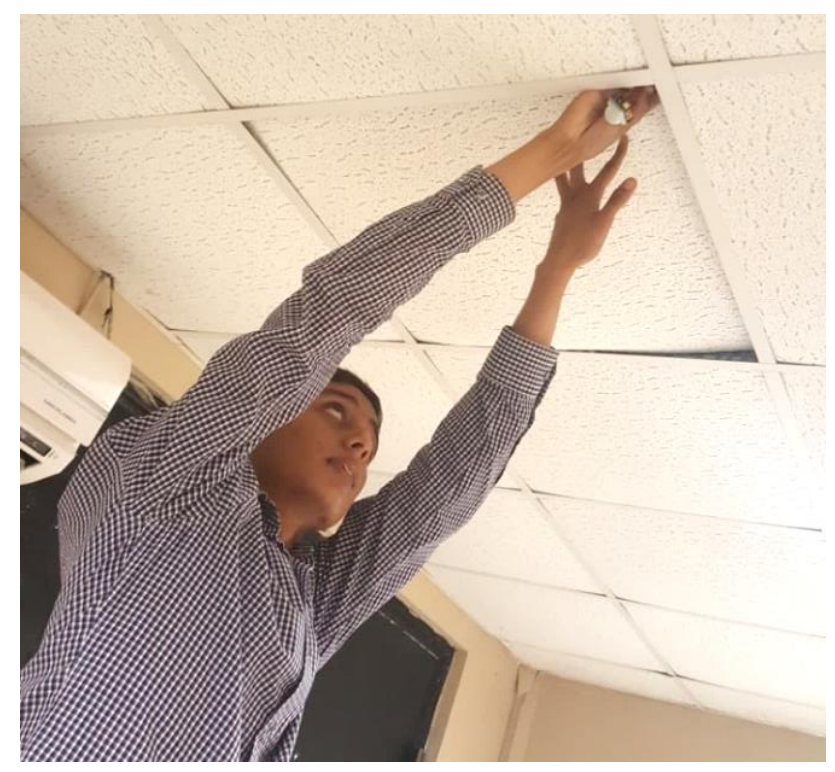

Figura 4 Interconexión clima y sensor de presencia

\section{Refrerencias}

Bastidas, C. A. y Medina, W. E. (2019). Construcción del prototipo de una planta para el control de procesos industriales, como apoyo en las prácticas del área de control de la Licenciatura en Electrónica (Tesis de grado). Universidad Pedagógica Nacional Facultad De Ciencia y Tecnología, Bogotá D.C., Colombia.

Gutiérrez, H. y De la Vara, R. (2012). Análisis y Diseño de Experimentos ( $3^{a}$ ed.). México: MCGRAW-HILL EDUCACION.

López, E. (2016). Arduino: Guía Práctica de Fundamentos y Simulación ( $1^{a}$ ed.). España: RaMa.
MINITAB (2019). Minitab®. Recuperado el 18 de enero del 2019, de: http://www.minitab.com/es-mx/

Montgomery, D. (2013). Diseño y Análisis de Experimentos ( $2^{a}$ ed.). México: LIMUSA WILEY.

Ogata, K. (2003). Ingeniería de Control Moderna ( $\left.4^{a} e d.\right)$. España: Pearson Educación. Palacios, E., Ramiro, F. \& López, L.J. (2015). Microcontrolador PIC16F84 ( $3^{a}$ ed.). México: Alfaomega.

Reyes, F. y Cid, J. (2015). Arduino. Aplicaciones en Robótica, Mecatrónica e Ingeniería ( $1^{a}$ ed.). México: Alfaomega.

Rivera, B. (2019). Método de proyecto y su influencia para desarrollar habilidades en instrumentación industrial de los estudiantes del tercer ciclo de electrónica industrial del instituto superior tecnológico público "Almirante Miguel Grau-Piura" (Tesis de grado). Universidad Católica de los Ángeles Chibote, Piura, Perú.

Rojas, J. S. y Contreras, S. (2019). Construcción de un prototipo para el mejoramiento de la enseñanza de la energía fotovoltaica (Trabajo de grado). Universidad Santo Tomás, Bogotá D.C., Colombia.

Torrente, O. (2013). Arduino: Curso Práctico de Formación (1 $1^{a} e d$.). México: Alfaomega.

Torres, P. S. y Pérez, F. R. (2019). Diseño y simulación de un módulo didáctico de un sistema de bombeo a presión constante para la capacitación de los alumnos de Ingeniería Electrónica de la UNPRG (Tesis de grado). Universidad Nacional "Pedro Ruiz Gallo", Lambayeque, Perú.

V.V.A.A. (2011). I.B.D.: Puesta en Servicio y Operación de Instalaciones Solares térmicas. ENAE0208 - Montaje y Mantenimiento de Instalaciones Solares Térmicas ( $1^{a}$ ed.). España: IC Editorial.

Valdés, F.E. \& Pallás R. (2016). Microcontroladores: Fundamentos y Aplicaciones con PIC $\left(22^{a}\right.$ ed.). México: Alfaomega.
GALARZA-SOSA, Oscar, MALDONADO-PESINA, Ericka, MARTINEZ-TOVAR, Cesar y GALINDO-CERDA, Herlinda. Aplicación de dispositivos electrónicos para el ahorro de energía eléctrica, en la materia de Instrumentación apoyando la docencia y aprendizaje. Revista de Tecnología y Educación. 2019 